\title{
A STABLE TIME DISCRETIZATION OF THE STEFAN PROBLEM WITH SURFACE TENSION*
}

\author{
BEN SCHWEIZER ${ }^{\dagger}$
}

\begin{abstract}
We present a time discretization for the single phase Stefan problem with GibbsThomson law. The method resembles an operator splitting scheme with an evolution step for the temperature distribution and a transport step for the dynamics of the free boundary. The evolution step involves only the solution of a linear equation that is posed on the old domain. We prove that the proposed scheme is stable in function spaces of high regularity. In the limit $\Delta t \rightarrow 0$ we find strong solutions of the continuous problem. This proves consistency of the scheme, and additionally it yields a new short-time existence result for the continuous problem.
\end{abstract}

Key words. free boundary problem, time discretization, operator splitting

AMS subject classifications. 35R35, 65M12, 80A22

PII. S003614290037232X

1. Introduction. The Stefan problem is a set of equations that describe the melting of ice or the growth of ice cristals. At time $t$ the ice (or the water) occupies a region $\Omega_{t}$, and the second phase occupies the complement of $\Omega_{t}$. The position of the interface $\partial \Omega_{t}$ is not known a priori but must be determined together with the temperature distribution $\Theta(t)$. Several sets of evolution equations can be found in the extensive literature (see [11] for equations and further references). Commonly used is the heat equation (2.1) in the domain $\Omega_{t}$ (in the two phase problem another heat equation is posed in the complement of $\Omega_{t}$ ). The latent heat relates the normal heat flux on the free boundary (or its jump across the boundary) with the speed of the free boundary as in (2.2). In order to determine the evolution we need one more boundary condition. Various possibilities are studied for that: (a) fixed temperature $\Theta=0$, (b) the Gibbs-Thomson relation $\Theta \sim \kappa$ with $\kappa$ being the mean curvature of the boundary, and (c) kinetic undercooling: temperature plus a multiple of the speed is proportional to the mean curvature. In the paper at hand we are interested in case (b), the Gibbs-Thomson relation (2.3).

The aim of this paper is to introduce a stable time discretization of the twodimensional free boundary value problem. We consider the single phase problem for simplicity; the two phase problem can be treated with the same method. Since the domain changes with time, it is not clear what equations we should pose at every time step, how to define a new domain, and how to define a temperature distribution on the new domain. Thinking of the numerical use of the scheme it is desirable that at each time step only a linear equation must be solved. This linear equation should be posed on the old domain. Our scheme will provide exactly this. As a by-product of our stability result in Theorem 2.2 we find a short-time existence result for (2.1)-(2.3) in Corollary 2.3. Such a result (in different function spaces) was proved earlier by Radkevich in [8]. Our approach is more elementary in the sense that it involves less functional-analytic machinery.

\footnotetext{
${ }^{*}$ Received by the editors May 11, 2000; accepted for publication (in revised form) March 12, 2002; published electronically September 12, 2002.

http://www.siam.org/journals/sinum/40-3/37232.html

${ }^{\dagger}$ Institut für Angewandte Mathematik, Im Neuenheimer Feld 294, D-69120 Heidelberg, Germany (ben.schweizer@iwr.uni-heidelberg.de).
} 
Another time discretization for the Gibbs-Thomson law was introduced by Luckhaus in [6]. His approach assumes only very low regularity such that solutions can be defined past geometric singularities. For that it is necessary to use explicitly the new domain in the definition of the time step. In this context we wish to mention the work of Bänsch [3] dealing with a time discretization for the Navier-Stokes equations with a free boundary. Also in these more complicated equations the new geometry is needed in the definition of the new iterate.

Let us compare the Gibbs-Thomson law (b) with kinetic undercooling (c). The term introduced in case (c) is regularizing; mathematically it has the effect that one can regard the equations as a coupled system of a heat equation and an equation for the motion of the free boundary. The regularity properties of the two evolution equations allow us to iterate the two solution operators. The fixed point is a solution of the original problem. Such an iteration is used by Chen and Reitich in [4] and by Abergel et al. in [1] in order to derive an existence result in case (c). A spatial semidiscretization was studied by Veeser in [10]. In contrast to case (c), it seems impossible to decouple the equations in case (b).

This paper is organized as follows. In section 2 we present the operator splitting scheme (OS) for a time discretization. Each time step consists of (1) defining an auxiliary velocity field $v,(2)$ solving a linear equation with transport term $v \cdot \nabla$, and (3) defining the new domain and a temperature field on the new domain by advection. In Theorem 2.2 and Corollary 2.3 we state our main result: the proposed scheme (OS) is stable and consistent.

Within this paper we introduce three different schemes. Scheme (OS) is the numerically applicable scheme in physical variables. The analysis of (OS) is the goal of this paper, and the results are collected in section 2 . In order to prove our results we introduce a linear Crank-Nicolson-type scheme $(\mathrm{CN})$ for unknowns $(u, h)$. (CN) is defined on a fixed domain and considers a given right-hand side $f$; detailed a priori estimates are derived in section 3 . The next step is to consider scheme $(\mathrm{CN})$ with a right-hand side of the form $f=f(u, h)$. Note that this is in general not a practical numerical scheme, since $f$ may depend on the values of the solution at later times. The special choice of $f(u, h)$ in section 4 is motivated by the original equations and their transformation to a fixed domain. We prove the existence of solutions and a priori estimates. In section 5 we conclude that the original scheme (OS) inherits these properties.

As already mentioned, our analysis is based on the study of a linear problem. This linear problem is obtained by transforming the equations onto a rectangle and linearizing them. This defines an operator in the unknown quantities temperature distribution $u$ and height function of the free boundary $h$. This linear operator has a compact inverse with regularizing properties. It allows us to solve instationary problems with a time discretization $(\mathrm{CN})$. The discretization can be proven to be stable by a testing procedure. Since the nonlinearity requires regular solutions, we apply the results also to discrete time derivatives and to second spatial derivatives of the time-discrete solutions. This yields estimates in function spaces of high regularity. In section 3 we collect estimates for $(\mathrm{CN})$, the semidiscrete equations on a fixed domain. Some care must be taken of compatibility conditions of the initial values.

Note that similar facts of the corresponding linearized problem were used in [9] in order to treat the Navier-Stokes equations with a free boundary. Let us again compare cases (b) and (c): in case (c) the properties of the linear operator can be shown with an iteration that solves successively for $u$ and $h$. In the case at hand one actually has to study the coupled system. 
In section 4 we consider a time discretization of transformed equations and apply the results of section 3. It turns out to be of importance in which point we linearize the equations. Concerning the mean curvature operator of the Gibbs-Thomson law it is sufficient to linearize it about the initial values. This is different for the nonlinearity introduced by the domain transformation: it has a different structure and cannot be treated by introducing error terms on the right-hand side (see Lemma 3.2 and remarks thereafter). We have to use in every time step the linearization of the equations on the current "old" domain. This introduces time-dependent coefficient matrices in the equations, but this way the transformation respects the variational character of the problem. As it turns out, the scheme (OS) mimics this strategy of linearization.

We encounter the fact that the solution of the discrete equations does not satisfy maximal regularity estimates. Therefore we have to be careful in the discretization of the nonlinearity.

In section 5 we prove Theorem 2.2 for scheme (OS). The idea is to transform the operator splitting scheme onto a reference domain and to apply the results of section 3 . It will turn out that the transformation of scheme (OS) is actually identical to the scheme of section 4 . The results of section 4 imply the stability of the transformed scheme and therefore the stability of the original scheme. Since (OS) is consistent with the continuous equations we can conclude that weak limits of the discrete solutions define strong solutions of the original problem.

2. The free boundary problem and the time discretization. We denote the domain that is covered with ice (or water) at time $t$ by $\Omega_{t}$. For notational convenience we assume that the free boundary is given as the graph of a single function. We study the two-dimensional case and write $S:=[0,1]_{\text {per }}$ for the unit interval with identified endpoints. A function defined on $S$ is automatically periodic; in particular, all derivatives (if defined) coincide in the endpoints. We write the domain as

$$
\Omega_{t}=\{(x, y) \mid x \in S, 0<y<h(t, x)\} .
$$

The height function $h$ will be close to 1 , and we can always parametrize $\Omega_{t}$ over the standard rectangle $S \times(0,1)$. Again, all functions on the rectangle are automatically periodic on the lateral boundaries. We introduce the time-dependent function $H(t, x)=(x, h(t, x))$ to parametrize the upper boundary of $\Omega_{t}$. In the following we will often omit the argument $t$. By a rescaling argument we can assume that the physical constants latent heat, surface tension, and thermal diffusion are all equal to 1. The physical equations then read

$$
\begin{aligned}
\partial_{t} \Theta & =\Delta \Theta & & \text { in } \bigcup_{t>0}\{t\} \times \Omega_{t}, \\
\partial_{t} h & =-(n \cdot \nabla \Theta) \circ H \sqrt{1+\left|\partial_{x} h\right|^{2}} & & \text { on }\{(t, x) \mid t>0, x \in S\}, \\
\Theta \circ H & =\kappa & & \text { on }\{(t, x) \mid t>0, x \in S\} .
\end{aligned}
$$

Here

$$
\kappa:=-\partial_{x}\left(\frac{\partial_{x} h}{\sqrt{1+\left|\partial_{x} h\right|^{2}}}\right)
$$

is the mean curvature of the free boundary, $n$ is the exterior normal of $\Omega$, and $n_{2}=$ $\left(1+\left|\partial_{x} h\right|^{2}\right)^{-1 / 2}$ the second component of $n$. The above equations are complemented with a boundary condition for $\Theta$ on the lower boundary, say,

$$
\Theta(t, x, 0)=\psi(t, x) .
$$


For notational convenience we will use $\psi \equiv 0$ in the following. All results remain valid for smooth $\psi$. Additionally, initial values $\Theta(t=0)=\Theta_{0}$ and $h(t=0)=h_{0}>0$ are imposed.

Later on we will use the linearization of the mean curvature operator about $h=$ $h_{0}$,

$$
\underline{\Delta} h:=-D \kappa\left(h_{0}\right) \cdot h=\partial_{x}\left(\frac{\partial_{x} h}{\sqrt{1+\left|\partial_{x} h_{0}\right|^{2}}}\right) .
$$

For smooth and small $h_{0}$ the properties of $\Delta$ are similar to those of $\Delta_{x}=\partial_{x}^{2}$, therefore the notation.

We now introduce a uniform discretization of the time interval $(0, T)$ by $t_{k}:=$ $k \cdot \Delta t$. Note that nonuniform time partitions can also be treated with our method. The pair $\left(\Theta^{k}, h^{k}\right)$ is meant to approximate $\left(\Theta\left(t_{k}\right), h\left(t_{k}\right)\right)$. We set $\left(\Theta^{0}, h^{0}\right):=\left(\Theta_{0}, h_{0}\right)$. The function $h^{k}$ defines the domain $\Omega^{k}:=\left\{(x, y) \mid x \in S, 0<y<h^{k}(x)\right\}$ and the normal vector $n^{k}$. We use $H^{k}(x):=\left(x, h^{k}(x)\right) \in \mathbb{R}^{2}$. In the following definition we need functions $\Theta^{(-1)}:=\tilde{\Theta}^{(-1)}:=\Theta^{0}$ for the first execution of Step 1. We define $H^{(-1)}$ and $n^{(-1)}$ via $h^{(-1)}:=h^{0}$.

Let us motivate in advance (2.5): let $\Theta$ solve $\partial_{t} \Theta=\Delta \Theta$ on the time-dependent domain $\Omega_{t}$. We consider $\tilde{\Theta}(t,):.=\Theta(t, \Phi(t,)$.$) , where \Phi(t,$.$) parametrizes \Omega_{t}$ over the fixed domain $\Omega_{t_{0}}: \Phi(t,):. \Omega_{t_{0}} \rightarrow \Omega_{t}$. Then $\Theta$ satisfies

$$
\partial_{t} \tilde{\Theta}=\left(\partial_{t} \Theta\right) \circ \Phi+(\nabla \Theta) \circ \Phi \cdot \partial_{t} \Phi=\left.\Delta \Theta\right|_{\Phi}+\left.\partial_{t} \Phi \cdot \nabla \Theta\right|_{\Phi} .
$$

If we want to calculate on a given domain (the "old" domain $\Omega_{t_{0}}$ ), then we have to include a convective term in the heat equation. In the numerical scheme it remains to choose a guess for the corresponding velocity field.

DeFinition 2.1. We assume that an initial domain $\Omega^{0}$ is given by $h^{0}$ and an initial temperature by $\Theta^{0}: \Omega^{0} \rightarrow \mathbb{R}$. Let $X^{0}: R \rightarrow \Omega^{0}$ be a parametrization of $\Omega^{0}$.

The operator splitting scheme (OS) for a time discretization of $(2.1)-(2.3)$ is defined by the following three steps; they are executed beginning with $k=0$.

Step 1 . We use the temperature data of the last time step in order to define a vertical velocity field $v^{k}=\left(v_{1}, v_{2}\right)=\left(0, v_{2}\right): \Omega^{k} \rightarrow \mathbb{R}^{2}$ with boundary values

$$
n^{k-1} \circ H^{k-1} \cdot v^{k} \circ H^{k}=\left(n^{k-1} \cdot \nabla \frac{\Theta^{k-1}+\tilde{\Theta}^{k-1}}{2}\right) \circ H^{k-1}
$$

by the linear interpolation

$$
v^{k}(x, y)=\frac{y}{h^{k}(x)} v^{k}\left(x, h^{k}(x)\right) .
$$

Step 2. Find $\tilde{\Theta}^{k}: \Omega^{k} \rightarrow \mathbb{R}$ and $h^{k+1}:[0,1] \rightarrow \mathbb{R}$ with

$$
\begin{array}{r}
\frac{\tilde{\Theta}^{k}-\Theta^{k}}{\Delta t}=\Delta\left(\frac{\Theta^{k}+\tilde{\Theta}^{k}}{2}\right)+v^{k} \cdot \nabla \frac{\Theta^{k}+\tilde{\Theta}^{k}}{2} \text { in } \Omega^{k}, \\
\frac{h^{k+1}-h^{k}}{\Delta t}=-\sqrt{1+\left|\partial_{x} h^{k}\right|^{2}}\left(n^{k} \cdot \nabla \frac{\Theta^{k}+\tilde{\Theta}^{k}}{2}\right) \circ H^{k} \text { in }[0,1], \\
\tilde{\Theta}^{k} \circ H^{k}+\underline{\Delta}\left(h^{k+1}-h^{k}\right)=\kappa\left(h^{k}\right) \text { in }[0,1] .
\end{array}
$$


On the lower boundary we impose $\tilde{\Theta}^{k}(x, 0)=\psi\left(x, t_{k}\right)$. We slightly change the definition in the first time step $k=0$. There we use $\Theta_{0}$ instead of $\frac{1}{2}\left(\Theta^{0}+\tilde{\Theta}^{0}\right)$ in the convective term of $(2.5)$.

Step 3 . The function $h^{k+1}$ defines the new domain $\Omega^{k+1}$. We now want to define a temperature field $\Theta^{k+1}$ on the new domain. We set

$$
\begin{aligned}
X^{k+1}(x, y) & :=X^{k}(x, y)+\left(0, \frac{X_{2}^{k}(x, y)}{h^{k}(x)}\right) \cdot\left(h^{k+1}-h^{k}\right)(x), \\
\Theta^{k+1} \circ X^{k+1} & :=\tilde{\Theta}^{k} \circ X^{k} \quad \text { in } R .
\end{aligned}
$$

We will show that the above scheme can be used to define uniquely $\left(\Theta^{k}, X^{k}\right)_{k=0, \ldots, K}$. The functions $\Theta^{k}$ are defined on domains that depend on time (on $k$ ). The domains are always parametrized by $X^{k}=\left(X_{1}^{k}, X_{2}^{k}\right)$. In order to formulate estimates we introduce the pairs $\left(u^{k}, h^{k}\right):=\left(\Theta^{k} \circ X^{k}, h^{k}\right)$. The functions $u^{k}$ are then defined on the time-independent domain $R$.

The main result of this paper is the following theorem. It is proved together with its corollary in section 5 .

THEOREM 2.2. Let the initial values $\left(u_{0}, h_{0}\right)$ satisfy the regularity and compatibility assumption, Assumption 5.1, and let $h_{0}-1$ be small in $C^{0,1}(S)$. Let the initial domain be parametrized over the rectangle $R=S \times(0,1)$ with a diffeomorphism $X^{0} \in H^{4+1 / 2}(R)$ with $X^{0}-i d$ small in $C^{0,1}(R), X_{1}^{0}(x, y)=x$ and $\partial_{2} X_{2}^{0}(., 1)=1$.

Then, on a small time interval $I=(0, T)$ the scheme $(O S)$ has a unique solution for $k=1, \ldots, K$ with $t_{K}<T$. The scheme is stable: the linear interpolant $(u, h)$ of $\left(u^{k}, h^{k}\right)_{k}$ satisfies the estimate

$$
\begin{aligned}
& \|h\|_{L^{\infty}\left(I ; H^{4+1 / 2}(S)\right)}+\|h\|_{W^{1, \infty}\left(I ; H^{2+1 / 2}(S)\right)} \\
& \quad+\|u\|_{L^{\infty}\left(I ; H^{3}(R)\right)}+\|u\|_{W^{1, \infty}\left(I ; H^{1}(R)\right)} \leq C .
\end{aligned}
$$

The number $C$ and the time interval I depend only on the initial values $\left(\Theta_{0}, h_{0}\right)$. They are independent of the time-step size $\Delta t$.

Corollary 2.3. Consider solutions $(u, X)_{\Delta t}$ as in Theorem 2.2. For a subsequence $\Delta t \rightarrow 0$ there holds

$$
(u, X)_{\Delta t} \longrightarrow(\tilde{u}, \tilde{X}) \quad \text { for } \quad \Delta t \rightarrow 0
$$

in the norms of $L^{2}\left(I ; H^{2}(R)\right) \cap H^{1}\left(I ; L^{2}(R)\right)$ and of $H^{1}\left(I ; H^{3}(R)\right)$. The limit function $(\Theta, X):=\left(\tilde{u} \circ \tilde{X}^{-1}, \tilde{X}\right)$ is a strong solution of the physical problem $(2.1)-(2.3)$.

Note that in the above results no smallness assumption is made on $\Theta_{0}$; the velocity of the boundary can be large, and convective effects must be included in the scheme. On the other hand, we assume smallness of $X^{0}$. This is not a severe restriction, since one could parametrize all domains $\Omega^{k}$ over a reference domain that is close to $\Omega^{0}$. Then smallness of $X^{0}$ is guaranteed.

A remark on implementations of the scheme. In the stability result we use the assumption that initially the height function is almost constant. This is done in order to simplify the proofs. It would be sufficient to have the initial domain close to a smooth reference domain (which is no restriction if the initial values are smooth).

Running the scheme is possible only for small times. This is because one of the following may happen: (1) The domain transformation onto the reference domain introduces large errors. (2) Using the linearization of the mean curvature operator about the initial values is no longer appropriate. (3) A geometric singularity makes a 
smooth parametrization impossible. Note that this is possible also for the continuous equations.

The best we can expect of the discretization is to work well as long as there exist continuous solutions of the system, that is, until problem (3) appears. In general, our method will fail to work before that time, due to problem (1) or (2). In this case one may continue with a restart: choose a new smooth reference domain, calculate the new linearized mean curvature operator, and restart the scheme.

3. A Crank-Nicolson scheme on the reference domain. After a transformation of (2.1)-(2.3) onto the reference domain $R=S \times(0,1)$ the equations have a linearization of the form (3.1)-(3.3). This section is devoted to the study of these linear equations on the rectangle.

$$
\begin{aligned}
\partial_{t} u & =\nabla \cdot A(t) \nabla u+f_{0} & & \text { in } R, \\
\partial_{t} h & =-a(t) \cdot \nabla u(., 1)+f_{1} & & \text { on } S, \\
u(., 1) & =-\underline{\Delta} h+f_{2} & & \text { on } S .
\end{aligned}
$$

We assume $a(t)=e_{2} \cdot A(t)$ and $A(t): R \rightarrow \mathbb{R}^{2 \times 2}$. In the following we always impose without further mentioning the condition $u=\psi=0$ (and $u^{k}=0$ ) on the lower boundary $\{(x, y) \mid y=0\}$. This also enables us to make use of the Poincaré inequality in what follows. A natural time discretization of (3.1)-(3.3) is the following CrankNicolson scheme.

DeFINITION 3.1. We denote the following scheme by (CN). In every time step we define $u^{k+1}: R \rightarrow \mathbb{R}, h^{k+1}: S \rightarrow \mathbb{R}$ as the solution of

$$
\begin{array}{ll}
\frac{u^{k+1}-u^{k}}{\Delta t}=\nabla \cdot A^{k} \nabla \frac{u^{k}+u^{k+1}}{2}+f_{0}^{k} & \text { in } R, \\
\frac{h^{k+1}-h^{k}}{\Delta t}=-a^{k} \cdot \nabla\left(\frac{u^{k}+u^{k+1}}{2}\right)(., 1)+f_{1}^{k} & \text { on } S, \\
u^{k+1}(., 1)=-\underline{\Delta} h^{k+1}+f_{2}^{k+1} & \text { on } S .
\end{array}
$$

Notation. In the following we will denote the averages of solutions at intermediate points as $u^{k+1 / 2}:=\frac{u^{k}+u^{k+1}}{2}$. The linear interpolant of the values $\left(u^{k}, h^{k}\right)$ will always be denoted by $(u, h)$, and linear interpolants of $f^{k}=\left(f_{0}^{k}, f_{1}^{k}, f_{2}^{k}\right)$ are denoted by $f=\left(f_{0}, f_{1}, f_{2}\right)$. We will once also use the linear interpolant of the values $u^{k+1 / 2}$; it will be denoted by $\bar{u}$.

In the scheme $(\mathrm{CN})$ the matrices $A^{k}$ will be uniformly close to the identity $I_{2} \in$ $\mathbb{R}^{2 \times 2}$. Nevertheless, it will be of importance to use the coefficient matrices in (3.4) and the corresponding oblique derivatives in (3.5). Loosely speaking, we must avoid any error term $f_{1}^{k}$ in (3.5). This statement is made precise in the subsequent lemma. The lemma gives a result on the resolvent problem corresponding to (3.1)-(3.3). It introduces function spaces that are natural for the problem.

LEMma 3.2 (the resolvent problem in energy spaces). Let $A: R \rightarrow \mathbb{R}^{2 \times 2}$ be a field of uniformly elliptic and symmetric matrices. Then for $\lambda>0$ the equations

$$
\begin{aligned}
\lambda u-\nabla \cdot A \nabla u & =g_{0} & & \text { in } R, \\
\lambda h+e_{2} \cdot A \cdot \nabla u(., 1) & =g_{1} & & \text { on } S, \\
u(., 1)+\Delta_{x} h & =g_{2} & & \text { on } S,
\end{aligned}
$$


together with $u(., 0)=0$, have a unique solution $(u, h)$. It satisfies the resolvent estimate

$$
\begin{array}{r}
\lambda^{2}\|u\|_{0}^{2}+\|\nabla \cdot A \nabla u\|_{0}^{2}+\lambda^{2} \int_{S}\left|\partial_{x} h\right|^{2}+\lambda\|h\|_{2+1 / 2}^{2} \\
\leq C\left(\left\|g_{0}\right\|_{0}^{2}+\left\|g_{1}\right\|_{1}^{2}+\lambda^{2}\left\|g_{2}\right\|_{-1}^{2}+\lambda\left\|g_{2}\right\|_{1 / 2}^{2}\right),
\end{array}
$$

with $C$ independent of $\lambda$. Here $\|\cdot\|_{s}$ denotes the norm of $H^{s}$.

Proof. To prove existence we assume $g_{2}=0$; this can be achieved by defining the new unknown to be $h-\Delta_{x}^{-1} g_{2}$. We find $u$ as the minimizer of

$$
\begin{aligned}
E(u):= & \lambda \int_{R} u^{2}+\int_{R} A \nabla u \cdot \nabla u-\lambda \int_{S} \Delta_{x}^{-1} u(., 1) \cdot u(., 1) \\
& -2 \int_{R} g_{0} \cdot u+2 \int_{S} g_{1} \cdot u(., 1)
\end{aligned}
$$

in $\left\{u \in H^{1}(R) \mid u(., 0)=0, \int_{S} u(., 1)=0\right\}$. Here the operator $\Delta_{x}^{-1}$ is defined by prescribing vanishing averages. With the function $\tilde{h}:=\Delta_{x}^{-1} u(., 1)$ the pair $(u, \tilde{h})$ solves (3.7), (3.9) exactly and (3.8) up to a constant function. Defining $h(x):=$ $\bar{h}+\tilde{h}(x)$ with an appropriate constant $\bar{h}$ we obtain a solution to $(3.7)-(3.9)$.

To find the a priori estimate we multiply (3.7) with $\lambda u-\nabla \cdot A \nabla u$ and integrate over $R$. This yields

$$
\lambda^{2} \int_{R}|u|^{2}+\int_{R}|\nabla \cdot A \nabla u|^{2}-2 \lambda \int_{R} u \nabla \cdot A \nabla u=\int_{R}(\lambda u-\nabla \cdot A \nabla u) g_{0} .
$$

With another integration by parts we find

$$
\begin{aligned}
& \lambda^{2} \int_{R}|u|^{2}+\int_{R}|\nabla \cdot A \nabla u|^{2}+2 \lambda \int_{R} \nabla u \cdot A \nabla u \\
& \quad+2 \lambda \int_{S}\left(g_{2}-\Delta_{x} h\right)\left(\lambda h-g_{1}\right)=\int_{R}(\lambda u-\nabla \cdot A \nabla u) g_{0} .
\end{aligned}
$$

The third term is positive, and in the fourth term we perform an integration by parts over $S$. We find an estimate for the first three terms on the left-hand side of (3.10). The estimate for $\sqrt{\lambda} h \in H^{2+1 / 2}(S)$ then follows from regularity for (3.9).

We read the above lemma as follows: the linearized problem has a good resolvent operator, and we can expect high regularity of solutions of the coupled problem. There are two restrictive points. In (3.10) an estimate of $\lambda g_{2}$ is needed on the right-hand side. This means that in the time-dependent problem the time derivative of $f_{2}$ must be controlled. The second difficulty is the regularity property that is assumed for $g_{1}$. In particular, we cannot insert an error of the form "trace of a first derivative of $u$." This is the reason why we use the oblique derivatives in (3.5).

Definition 3.3. For a solution $(u, h)$ we define the Banach space $Y:=Y_{u} \times Y_{h}$ with

$$
\begin{aligned}
& Y_{u}:=L^{\infty}\left(0, T ; H^{1}(R)\right) \cap H^{1}\left(0, T ; L^{2}(R)\right), \\
& Y_{h}:=L^{\infty}\left(0, T ; H^{2+1 / 2}(S)\right) \cap H^{1}\left(0, T ; H^{1}(S)\right) .
\end{aligned}
$$

To control the right-hand side we define the Banach spaces

$$
\begin{aligned}
& X_{0}:=L^{2}\left(0, T ; L^{2}(R)\right), \\
& X_{1}:=L^{2}\left(0, T ; H^{1}(S)\right), \\
& X_{2}:=L^{\infty}\left(0, T ; H^{1 / 2}(S)\right) \cap H^{1}\left(0, T ; H^{-1}(S)\right) .
\end{aligned}
$$


Observe that the above are not the maximal regularity spaces of the continuous equations. For that we would expect additional estimates for $u \in L^{2}\left(I ; H^{2}\right)$ and $h \in L^{2}\left(I ; H^{3+1 / 2}\right)$. However, the above Crank-Nicolson scheme will not provide such an estimate. It can provide it at best for the interpolant of the midpoints $\frac{1}{2}\left(u^{k}+u^{k+1}\right)$.

LEMMA 3.4 (the scheme (CN) in energy spaces). Assume that the coefficient matrices $A^{k}$ in Definition 3.1 are symmetric and satisfy

$$
\sup _{k}\left\|A^{k}-I_{2}\right\|_{L^{\infty}(R)}+\sum_{k}\left\|\frac{A^{k+1}-A^{k}}{\Delta t}\right\|_{L^{\infty}(R)} \Delta t<\delta .
$$

We consider initial values $u_{0} \in H^{1}(R), h_{0} \in H^{2+1 / 2}(S)$. Let (3.6) be satisfied for the initial values $\left(u^{0}, h^{0}\right):=\left(u_{0}, h_{0}\right)$; that is, (3.6) holds for $k=-1$. Given a righthand side $\left(f^{k}\right)_{k}$ we will write estimates in terms of the linear interpolant $f: I \rightarrow$ $L^{2}(R)^{2} \times L^{2}(S) \times L^{2}(S)$. Let the time interval $I=(0, T)$ and $\delta>0$ be small enough.

Then for every $K \in \mathbb{N}$ with $K \cdot \Delta t \leq T$ the linear scheme (CN) of Definition 3.1 has a unique solution $\left(u^{k}, h^{k}\right)_{k=0, \ldots, K}$. The linear interpolant $(u, h)$ of $\left(u^{k}, h^{k}\right)_{k}$ satisfies the estimate

$$
\|(u, h)\|_{Y} \leq C_{1}\left\|u_{0}\right\|_{H^{1}(R)}+C_{2}\left(\left\|f_{0}\right\|_{X_{0}}+\left\|f_{1}\right\|_{X_{1}}+\left\|f_{2}\right\|_{X_{2}}\right)
$$

with $C_{1}$ and $C_{2}$ independent of $\Delta t$.

The estimate (3.12) can be improved: on the right-hand side we can replace $\left\|f_{2}\right\|_{X_{2}}$ by $\left\|f_{2}\right\|_{H^{1}\left(0, T ; H^{-1}(S)\right)}+C_{\star}$, where $C_{\star}$ has the property that for some $C>0$ every solution of (3.6) satisfies

$$
\left\|h^{k+1}\right\|_{H^{2+1 / 2}(S)} \leq C_{\star}+C\left\|u^{k+1}\right\|_{H^{1}(R)} .
$$

Proof. The proof of this lemma relies on a testing procedure; it is analogous to the proof of the resolvent estimate of Lemma 3.2. We multiply (3.4) with $-\nabla$. $A^{k} \nabla\left(\frac{u^{k}+u^{k+1}}{2}\right)$. An integration over $R$ yields

$$
\begin{aligned}
& \int_{R} \nabla \frac{u^{k+1}-u^{k}}{\Delta t} \cdot A^{k} \nabla \frac{u^{k}+u^{k+1}}{2}+\left\|\nabla \cdot A^{k} \nabla \frac{u^{k}+u^{k+1}}{2}\right\|_{L^{2}(R)}^{2} \\
& -\int_{S} a^{k} \cdot \nabla\left(\frac{u^{k}+u^{k+1}}{2}\right) \frac{u^{k+1}-u^{k}}{\Delta t}=-\int_{R} f_{0}^{k} \cdot \nabla \cdot A^{k} \nabla \frac{u^{k}+u^{k+1}}{2} .
\end{aligned}
$$

We use the symmetry of $A^{k}$ to calculate for the first term

$$
\begin{aligned}
\int_{R} & \nabla \frac{u^{k+1}-u^{k}}{\Delta t} \cdot A^{k} \nabla \frac{u^{k}+u^{k+1}}{2} \\
& =\frac{1}{2 \Delta t} \int_{R} A^{k} \nabla u^{k+1} \cdot \nabla u^{k+1}-\frac{1}{2 \Delta t} \int_{R} A^{k} \nabla u^{k} \cdot \nabla u^{k} .
\end{aligned}
$$

To evaluate the boundary integral we use (3.5) with index $k$ and (3.6) with the indices $k$ and $k+1$ :

$$
\begin{aligned}
\int_{S} a^{k} \cdot \nabla & \left(\frac{u^{k}+u^{k+1}}{2}\right)\left(\frac{u^{k+1}-u^{k}}{\Delta t}\right) \\
= & \int_{S}\left(\frac{h^{k+1}-h^{k}}{\Delta t}-f_{1}^{k}\right) \cdot\left(\Delta \frac{h^{k+1}-h^{k}}{\Delta t}-\frac{f_{2}^{k+1}-f_{2}^{k}}{\Delta t}\right) .
\end{aligned}
$$


Inserting this into (3.13) we find

$$
\begin{aligned}
& \frac{1}{2 \Delta t} \int_{R} A^{k+1} \nabla u^{k+1} \cdot \nabla u^{k+1}-\frac{1}{2 \Delta t} \int_{R} A^{k} \nabla u^{k} \cdot \nabla u^{k} \\
& +\left\|\nabla \cdot A^{k} \nabla \frac{u^{k}+u^{k+1}}{2}\right\|_{L^{2}(R)}^{2}-\int_{S} \frac{h^{k+1}-h^{k}}{\Delta t} \cdot \Delta \frac{h^{k+1}-h^{k}}{\Delta t} \\
& =-\int_{R} f_{0}^{k} \cdot \nabla \cdot A^{k} \nabla \frac{u^{k}+u^{k+1}}{2}+\int_{R} \frac{A^{k+1}-A^{k}}{2 \Delta t} \nabla u^{k+1} \cdot \nabla u^{k+1} \\
& -\int_{S}\left(\frac{h^{k+1}-h^{k}}{\Delta t}-f_{1}^{k}\right) \cdot \frac{f_{2}^{k+1}-f_{2}^{k}}{\Delta t}-\int_{S} f_{1}^{k} \cdot \Delta \frac{h^{k+1}-h^{k}}{\Delta t} .
\end{aligned}
$$

Multiplication with $\Delta t$ and summing up over $k=0, \ldots, K-1$ we find

$$
\begin{aligned}
& \int_{R} A^{K} \nabla u^{K} \cdot \nabla u^{K}+\sum_{k}\left\|\nabla \cdot A^{k} \nabla \frac{u^{k}+u^{k+1}}{2}\right\|_{L^{2}(R)}^{2} \Delta t \\
& +\sum_{k} \int_{S}\left|\partial_{x}\left(\frac{h^{k+1}-h^{k}}{\Delta t}\right)\right|^{2} \Delta t \leq 2\left\|\nabla u^{0}\right\|_{L^{2}(R)}^{2} \\
& +2 \sum_{k}\left\|\frac{A^{k+1}-A^{k}}{\Delta t}\right\|_{L^{\infty}(R)} \cdot\left\|\nabla u^{k+1}\right\|_{L^{2}(R)}^{2} \Delta t \\
& +C \sum_{k}\left\{\left\|f_{0}^{k}\right\|_{0}^{2}+\int_{S}\left[\left|\partial_{x} f_{1}^{k}\right|^{2}+\left|f_{1}^{k}\right|^{2}\right]+\left\|\frac{f_{2}^{k+1}-f_{2}^{k}}{\Delta t}\right\|_{-1}^{2}\right\} \Delta t .
\end{aligned}
$$

For the linear interpolant $(u, h)$ of the sequence $\left(u^{k}, h^{k}\right)$ we find with (3.4) the estimate

$$
\|u\|_{L^{\infty}\left(I ; H^{1}(R)\right)}+\left\|\partial_{t} u\right\|_{L^{2}\left(I ; L^{2}(R)\right)}+\left\|\partial_{x} \partial_{t} h\right\|_{L^{2}\left(I ; L^{2}(S)\right)} \leq C .
$$

It remains to prove spatial regularity properties of $h$. Since traces of $u^{k}$ are bounded in the space $l^{\infty}\left(\{0, \ldots, K\} ; H^{1 / 2}(S)\right)$, (3.6) implies the regularity of $h$. The improved version of the estimate mimics this argument.

The nonlinearity of the original problem requires the control of the domain in regular norms. Estimates of higher order can be derived by considering derivatives of solutions. They satisfy again equations of the type (3.4)-(3.6), and we can apply Lemma 3.4.

We introduce a notation. As before we write $g$ for the linear interpolant of a set of functions $\left(g^{k}\right)_{k}$. We will write $\bar{\partial}_{t} g$ for the linear interpolant of the discrete time derivatives $\frac{g^{k}-g^{k-1}}{\Delta t}$. In this way we can use also time derivatives of $\bar{\partial}_{t} g$; they are piecewise constant functions with values $\frac{g^{k+1}-2 g^{k}+g^{k-1}}{(\Delta t)^{2}}$. The function $\bar{\partial}_{t} g$ is defined on the time interval $(\Delta t, T)$, and all norms are calculated on that interval. 
Proposition 3.5 (the scheme (CN) with higher regularity). Let the compatibility assumption, Assumption 3.6, on the initial values be satisfied. Assume that the coefficient matrices are symmetric and satisfy

$$
\begin{array}{r}
\sup _{k}\left\|A^{k}-I_{2}\right\|_{C^{0}(\bar{R})}<\delta, \\
\sum_{k}\left\{\left\|\nabla A^{k}\right\|_{H^{2}(R)}^{2}+\left\|\nabla A^{k}(., 1)\right\|_{H^{2}(S)}^{2}\right\} \Delta t<\delta^{2}, \\
\sum_{k}\left\{\left\|\frac{A^{k+1}-A^{k}}{\Delta t}\right\|_{H^{1}(R) \cap L^{\infty}(R)}^{2}+\left\|\frac{A^{k+1}-A^{k}}{\Delta t}(., 1)\right\|_{H^{1}(S)}^{2}\right\} \Delta t<\delta^{2} .
\end{array}
$$

On the initial values we assume $\left\|h_{0}-1\right\|_{C^{0,1}(S)}<\delta$. Let $T>0$ and $\delta>0$ be small enough and $\left(u^{k}, h^{k}\right)_{k}$ be a solution of scheme $(C N)$. Then the linear interpolant $(u, h)$ satisfies

$$
\begin{aligned}
&\left\|\bar{\partial}_{t}(u, h)\right\|_{Y}+\left\|\partial_{x}^{2}(u, h)\right\|_{Y} \\
& \leq C_{1}\left[\left\|\nabla \cdot A(0) \nabla u_{0}+f_{0}(0)\right\|_{H^{1}(R)}+\left\|\partial_{x}^{2} u_{0}\right\|_{H^{1}(R)}\right] \\
&+C_{2}\left[\left\|\bar{\partial}_{t} f_{0}\right\|_{X_{0}}+\left\|\bar{\partial}_{t} f_{1}\right\|_{X_{1}}+\left\|\bar{\partial}_{t} f_{2}\right\|_{X_{2}}\right] \\
&+C_{3}\left[\left\|\partial_{x}^{2} f_{0}\right\|_{X_{0}}+\left\|\partial_{x}^{2} f_{1}\right\|_{X_{1}}+\left\|\partial_{x}^{2} f_{2}\right\|_{X_{2}}\right] \\
&+C_{4} \delta\left[\left\|f_{0}\right\|_{L^{\infty}\left(I ; H^{1}(R)\right)}+\|A\|_{L^{\infty}\left(I ; H^{2}(R)\right)}\right] .
\end{aligned}
$$

The linear interpolant $\bar{u}$ of the midpoint values $u^{k+1 / 2}$ satisfies additionally the regularity estimate

$$
\|\bar{u}\|_{L^{\infty}\left(I ; H^{3}(R)\right)} \leq C_{5}\left(c_{0}+\left\|f_{0}\right\|_{L^{\infty}\left(I ; H^{1}(R)\right)}+\sup _{k}\left\|A^{k}\right\|_{H^{2}(R)}\right),
$$

where $c_{0}$ denotes the right-hand side of (3.17).

Proof. The assumptions on $A$ are stronger than those in Lemma 3.4. In particular, we know that a unique discrete solution exists on a small time interval and that it satisfies the estimate (3.12).

Part I. Time derivatives. We introduce discrete derivatives

$$
\tilde{u}^{k}:=\frac{u^{k}-u^{k-1}}{\Delta t}, \quad \tilde{h}^{k}:=\frac{h^{k}-h^{k-1}}{\Delta t}
$$

for all $k=1, \ldots, K$. We now use the definition of $\left(u^{k}, h^{k}\right)$ in (3.4)-(3.6). Taking the equations with index $k$ and subtracting the equations with index $k-1$ yields for the new functions the following set of equations:

$$
\begin{aligned}
& \frac{\tilde{u}^{k+1}-\tilde{u}^{k}}{\Delta t}=\nabla \cdot A^{k} \nabla \frac{\tilde{u}^{k}+\tilde{u}^{k+1}}{2}+\frac{f_{0}^{k}-f_{0}^{k-1}}{\Delta t} \\
&+\nabla \cdot\left(\frac{A^{k}-A^{k-1}}{\Delta t} \nabla \frac{u^{k-1}+u^{k}}{2}\right), \\
& \frac{\tilde{h}^{k+1}-\tilde{h}^{k}}{\Delta t}=- a^{k} \cdot \nabla\left(\frac{\tilde{u}^{k}+\tilde{u}^{k+1}}{2}\right)(., 1)+\frac{f_{1}^{k}-f_{1}^{k-1}}{\Delta t} \\
& \quad-\frac{a^{k}-a^{k-1}}{\Delta t} \cdot \nabla\left(\frac{u^{k-1}+u^{k}}{2}\right)(., 1), \\
& \tilde{u}^{k+1}(., 1)=-\Delta \tilde{h}^{k+1}+\frac{f_{2}^{k+1}-f_{2}^{k}}{\Delta t} .
\end{aligned}
$$


We read these equations as follows: $\left(\tilde{u}^{k}, \tilde{h}^{k}\right)_{k=1, \ldots, K}$ is a solution of the scheme $(\mathrm{CN})$ of Definition 3.1 with initial values $\left(\tilde{u}^{1}, \tilde{h}^{1}\right)$. The right-hand side is

$$
\begin{aligned}
& \tilde{f}_{0}^{k}:=\frac{f_{0}^{k}-f_{0}^{k-1}}{\Delta t}+\nabla \cdot\left(\frac{A^{k}-A^{k-1}}{\Delta t} \nabla \frac{u^{k}+u^{k-1}}{2}\right), \\
& \tilde{f}_{1}^{k}:=\frac{f_{1}^{k}-f_{1}^{k-1}}{\Delta t}-\frac{a^{k}-a^{k-1}}{\Delta t} \cdot \nabla\left(\frac{u^{k}+u^{k-1}}{2}\right)(., 1), \\
& \tilde{f}_{2}^{k}:=\frac{f_{2}^{k}-f_{2}^{k-1}}{\Delta t}
\end{aligned}
$$

for $k=1, \ldots, K$. We next apply Lemma 3.4 on the sequence $\left(\tilde{u}^{k}, \tilde{h}^{k}\right)_{k}$. We recall the notation $(\tilde{u}, \tilde{h})$ for the linear interpolant of $\left(\tilde{u}^{k}, \tilde{h}^{k}\right)_{k}$ and introduce $\tilde{f}_{i}$ for the linear interpolant of $\left(\tilde{f}_{i}^{k}\right)_{k}$. Note that the domain of definition is $(\Delta t, T)$; on this time interval we have by Lemma 3.4

$$
\|(\tilde{u}, \tilde{h})\|_{Y} \leq c_{0}\left\|\tilde{u}^{1}\right\|_{H^{1}(R)}+c_{1}\left[\left\|\tilde{f}_{0}\right\|_{X_{0}}+\left\|\tilde{f}_{1}\right\|_{X_{1}}+\left\|\tilde{f}_{2}\right\|_{X_{2}}\right] .
$$

The discrete time derivatives $\bar{\partial}_{t} f_{i}$ of $f_{i}$ enter the bound (3.17) explicitly. It remains to estimate the contributions

$$
\begin{aligned}
& \nabla \cdot\left(\frac{A^{k}-A^{k-1}}{\Delta t} \nabla \frac{u^{k}+u^{k-1}}{2}\right) \in X_{0}, \\
& \frac{a^{k}-a^{k-1}}{\Delta t} \cdot \nabla\left(\frac{u^{k}+u^{k-1}}{2}\right)(., 1) \in X_{1} .
\end{aligned}
$$

We find $c>0$ such that

$$
\begin{aligned}
& \sum_{k}\left\|\nabla \cdot\left(\frac{A^{k}-A^{k-1}}{\Delta t} \nabla \frac{u^{k}+u^{k-1}}{2}\right)\right\|_{L^{2}(R)}^{2} \Delta t \\
& \quad \leq c \sum_{k}\left\|\frac{A^{k}-A^{k-1}}{\Delta t}\right\|_{H^{1}(R)}^{2} \Delta t \cdot \sup _{k}\left\|\nabla \frac{u^{k}+u^{k-1}}{2}\right\|_{H^{2}(R)}^{2}, \\
& \sum_{k}\left\|\frac{a^{k}-a^{k-1}}{\Delta t} \cdot \nabla\left(\frac{u^{k}+u^{k-1}}{2}\right)(., 1)\right\|_{H^{1}(S)}^{2} \Delta t \\
& \quad \leq c \sum_{k}\left\|\frac{a^{k}-a^{k-1}}{\Delta t}(., 1)\right\|_{H^{1}(S)}^{2} \Delta t \cdot \sup _{k}\left\|\nabla \frac{u^{k}+u^{k-1}}{2}(., 1)\right\|_{H^{1}(S)}^{2} .
\end{aligned}
$$

With the assumptions on $A$ and $f_{i}$ Lemma 3.4 yields for $\bar{\partial}_{t}(u, h)=(\tilde{u}, \tilde{h})$ the estimate

$$
\left\|\bar{\partial}_{t}(u, h)\right\|_{Y} \leq c_{0}\left\|\tilde{u}^{1}\right\|_{H^{1}(R)}+c_{1} \delta \sup _{k}\left\|\frac{u^{k}+u^{k-1}}{2}\right\|_{H^{3}(R)}+c_{2},
$$

where $c_{2}$ depends only on the norms of $\bar{\partial}_{t} f_{i}$.

In order to treat the second term on the right-hand side we now show estimate (3.18). This is done with the help of the original equation (3.4). The elliptic equation with the boundary condition (3.5) yields the estimate

$$
\begin{aligned}
\left\|u^{k+1 / 2}\right\|_{H^{3}(R)} \leq C & \left(\left\|f_{0}^{k}\right\|_{H^{1}(R)}+\left\|f_{1}^{k}\right\|_{H^{3 / 2}(S)}\right. \\
& \left.+\left\|\bar{\partial}_{t}(u, h)\right\|_{Y}+\left\|A^{k}\right\|_{H^{2}(R)}\right) .
\end{aligned}
$$


Here the norm of $f_{1}$ is controlled by the right-hand side of (3.17). We have $\bar{\partial}_{t} f_{1}$ bounded in $L^{2}\left(0, T ; H^{1}(S)\right)$ and $f_{1}$ bounded in $L^{2}\left(0, T ; H^{3}(S)\right)$. An interpolation yields an estimate in $L^{\infty}\left(0, T ; H^{3 / 2}(S)\right)$ for $f_{1}$,

$$
\sup _{k}\left\|f_{1}^{k}\right\|_{H^{3 / 2}(S)} \leq C\left\{\left\|\partial_{t} f_{1}\right\|_{X_{1}}+\left\|\partial_{x}^{2} f_{1}\right\|_{X_{1}}\right\} .
$$

Equation (3.18) is shown.

We now insert (3.18) into estimate (3.24) and find with new constants $c_{0}, c_{1}$, and $c_{2}$,

$$
\begin{aligned}
\left\|\bar{\partial}_{t}(u, h)\right\|_{Y} \leq c_{0}\left\|\tilde{u}^{1}\right\|_{H^{1}(R)} \\
+c_{1} \delta\left(\left\|f_{0}\right\|_{L^{\infty}\left(I ; H^{1}(R)\right)}+\|A\|_{L^{\infty}\left(I ; H^{2}(R)\right)}\right)+c_{2},
\end{aligned}
$$

where $c_{2}$ depends only on the norms of $\bar{\partial}_{t} f_{i}$.

Part II. Spatial derivatives. Estimate (3.25) does not suffice for the analysis of the nonlinear problem. Note that the best spatial estimate for the boundary so far is $h \in C^{\alpha}\left(I ; H^{2+1 / 2}\right)$. We next want to derive an estimate for $h \in L^{\infty}\left(I ; H^{4+1 / 2}(S)\right)$ to have good control of the regularity of the boundary. This estimate could be derived from an estimate for $u \in L^{\infty}\left(I ; H^{3}(R)\right)$. A similar estimate does appear in (3.18) but only for interpolants of $\frac{1}{2}\left(u^{k}+u^{k+1}\right)$ and not for interpolants of $u^{k}$. In order to derive the regularity estimate on $h$ we perform an analysis of second spatial derivatives of the semidiscrete solution. While we used discrete derivatives in Part I we can now use classical derivatives. We introduce

$$
\hat{u}^{k}:=\partial_{x}^{2} u^{k}, \quad \hat{h}^{k}:=\partial_{x}^{2} h^{k} .
$$

As in Part I we will use the fact that $\left(\hat{u}^{k}, \hat{h}^{k}\right)_{k}$ is a solution of scheme (CN) for an appropriate right-hand side. To be precise, $\left(\hat{u}^{k}, \hat{h}^{k}\right)_{k}$ satisfies (3.4)-(3.6) with $\left(f_{0}^{k}, f_{1}^{k}, f_{2}^{k}\right)_{k}$ replaced by $\left(\hat{f}_{0}^{k}, \hat{f}_{1}^{k}, \hat{f}_{2}^{k}\right)_{k}$, defined by

$$
\begin{gathered}
\hat{f}_{0}^{k}:=\partial_{x}^{2} f_{0}^{k}+\nabla \cdot\left(\left[\partial_{x}^{2}, A^{k}\right] \nabla \frac{u^{k}+u^{k+1}}{2}\right), \\
{\left[\partial_{x}^{2}, A^{k}\right] w=\left(\partial_{x}^{2} A^{k}\right) w+2\left(\partial_{x} A^{k}\right) \partial_{x} w \quad \forall w,} \\
\hat{f}_{1}^{k}:=\partial_{x}^{2} f_{1}^{k}-\left[\partial_{x}^{2}, a^{k}\right] \cdot \nabla \frac{u^{k}+u^{k+1}}{2}, \\
{\left[\partial_{x}^{2}, a^{k}\right] \cdot w=\left(\partial_{x}^{2} a^{k}\right) w+2\left(\partial_{x} a^{k}\right) \partial_{x} w \quad \forall w,} \\
\hat{f}_{2}^{k}:=\partial_{x}^{2} f_{2}^{k}-\partial_{x}\left(\left[\partial_{x}^{2}, \gamma_{0}\right] \partial_{x} h^{k}\right), \\
{\left[\partial_{x}^{2}, \gamma_{0}\right] w=\left(\partial_{x}^{2} \gamma_{0}\right) w+2 \partial_{x} \gamma_{0} \partial_{x} w \quad \forall w,}
\end{gathered}
$$

where we introduced the abbreviation

$$
\gamma_{0}=\frac{1}{{\sqrt{1+\left|h_{0}^{\prime}\right|^{3}}}^{3}}
$$

We now use Lemma 3.4. With the notation $I_{K}=\{0, \ldots, K\}$ and $\bar{u}^{k}:=\frac{u^{k}+u^{k+1}}{2}$ we have to show estimates for

$$
\begin{array}{r}
\nabla \partial_{x}^{2} A^{k} \cdot \nabla \bar{u}^{k}, \nabla \partial_{x} A^{k} \cdot \nabla \partial_{x} \bar{u}^{k}, \\
\partial_{x}^{2} A^{k} \cdot \Delta \bar{u}^{k}, \partial_{x} A^{k} \cdot \Delta \partial_{x} \bar{u}^{k} \in l^{2}\left(I_{K} ; L^{2}(R)\right), \\
\partial_{x}^{2} a^{k} \cdot \nabla \bar{u}^{k}(., 1), \partial_{x} a^{k} \cdot \nabla \partial_{x} \bar{u}^{k}(., 1) \in l^{2}\left(I_{K} ; H^{1}(S)\right),
\end{array}
$$


and additionally estimates in $l^{2}\left(I_{K} ; H^{-1 / 2}(S)\right)$ for the discrete time derivatives of the functions

$$
\partial_{x}^{4} h_{0} \cdot \partial_{x} h^{k}, \partial_{x}^{3} h_{0} \cdot \partial_{x}^{2} h^{k}, \partial_{x}^{2} h_{0} \cdot \partial_{x}^{3} h^{k} .
$$

On the functions of the last line we additionally have to give an estimate in $l^{\infty}\left(I_{K} ; H^{1 / 2}(S)\right)$ or we use the improved version of estimate (3.12). We use the latter and see from the original equation (3.6) for $h^{k+1}$ that we can use $C_{\star}=$ $C\left\|f_{2}^{k}\right\|_{H^{2+1 / 2}(S)} \leq C\left(\left\|\partial_{x}^{2} f_{2}\right\|_{X_{2}}+\left\|\partial_{t} f_{2}\right\|_{X_{2}}\right)$, where $C$ depends only on $\left\|h_{0}\right\|_{H^{4+1 / 2}(S)}$. All the above error terms can be estimated by a small multiple of the solution norm in (3.17). While the other terms can be estimated directly, the most intricate term is the one containing second derivatives of the trace of derivatives of $\bar{u}^{k}$. It suffices to estimate for the interpolation

$$
\partial_{x}^{2} \nabla \bar{u}(., 1) \in L^{2}\left(I ; L^{2}(S)\right)
$$

by the norms of $u$ and $f$ in (3.17). This estimate can be derived from (3.4) if we differentiate that equation twice with respect to $x$. We use $\partial_{t} \partial_{x}^{2} u, \partial_{x}^{2} f_{0} \in L^{2}\left(I ; L^{2}(R)\right)$, and, for the boundary condition, $\partial_{t} \partial_{x}^{2} h \in L^{2}\left(I ; H^{1}(S)\right)$. Elliptic theory yields $\partial_{x}^{2} \bar{u} \in$ $L^{2}\left(I ; H^{2}(R)\right)$ and therefore the result.

We can now apply Lemma 3.4 which yields the $Y$-estimates for $\partial_{x}^{2}(u, h)$. The compatibility condition ((3.6) is satisfied for $k=-1)$ holds, since we took only second derivatives on both sides. Note that without the estimates of the time derivative we could not have derived the spatial estimates on $\bar{u}$ but only estimates on higher $x$ derivatives of $u$.

Part III. The first time step. It remains to control the first discrete time derivative $\tilde{u}^{1} \in H^{1}(R)$ of (3.25) by the first term on the right-hand side of (3.17). This is done in the subsequent lemma which concludes the proof of the proposition.

Assumption 3.6. We assume that $A=A(0)$ is a $\operatorname{Sym}\left(\mathbb{R}^{2}\right)$-valued function of class $H^{3}(R)$, sufficiently close to the identity in $L^{\infty}(R)$.

The compatibility conditions for the discrete scheme read

$$
\begin{aligned}
u_{0}(., 1) & =-\underline{\Delta} h_{0}+f_{2}^{0}, \\
\left(\nabla \cdot A \nabla u_{0}+f_{0}^{0}\right)(., 1) & =\underline{\Delta}\left(a \cdot \nabla u_{0}(., 1)-f_{1}^{0}\right)+\frac{f_{2}^{1}-f_{2}^{0}}{\Delta t} .
\end{aligned}
$$

Lemma 3.7. Let Assumption 3.6 be satisfied. Then the solution $\left(u^{1}, h^{1}\right)$ for the first time step in scheme (CN) satisfies

$$
\left\|\frac{u^{1}-u^{0}}{\Delta t}\right\|_{H^{1}(R)} \leq C\left\|\nabla \cdot A \nabla u_{0}+f_{0}\right\|_{H^{1}(R)}
$$

with $C$ independent of $\Delta t$.

Proof. We write $\underline{\Delta}=\partial_{x}\left(\gamma_{0} \partial_{x}\right)$ with $\gamma_{0}$ close to 1 in $L^{\infty}(S)$. We use $a=e_{2} \cdot A$ and study the operator

$$
B:\left(\begin{array}{l}
u \\
h
\end{array}\right) \mapsto\left(\begin{array}{c}
\nabla \cdot A \nabla u \\
-a \cdot \nabla u(., 1)
\end{array}\right)
$$

defined on

$$
D(B):=\left\{(u, h) \in X_{0} \mid u \in H^{2}(R), u(., 1)=-\underline{\Delta} h, u(., 0)=0\right\},
$$


a subset of the space

$$
X_{0}:=\left\{(u, h) \mid \int_{0}^{1} h=0\right\} \subset X:=L^{2}(R) \times H^{1}(S) .
$$

On $X_{0}$ we use the scalar product

$$
\left\langle\left(\begin{array}{l}
u \\
h
\end{array}\right),\left(\begin{array}{l}
\hat{u} \\
\hat{h}
\end{array}\right)\right\rangle:=\int_{R} u \cdot \hat{u}+\int_{S} \gamma_{0} \partial_{x} h \cdot \partial_{x} \hat{h} .
$$

Then the operator $B$ is densely defined in $X_{0}$, it has a compact inverse by Lemma 3.2, and it is symmetric. By the spectral theorem we find a complete set of eigenfunctions $\left(\sigma^{j}, \eta^{j}\right)$ of $B$; that is,

$$
\begin{aligned}
\lambda_{j} \sigma^{j}-\nabla \cdot A \nabla \sigma^{j} & =0, \\
\lambda_{j} \eta^{j}+a \cdot \nabla \sigma^{j}(., 1) & =0, \\
\sigma^{j}(., 1)+\Delta \eta^{j} & =0 .
\end{aligned}
$$

In order to have a basis $\left(\sigma^{j}, \eta^{j}\right)$ of $X$ (and not only on $X_{0}$ ) we extend the basis by eigenfunctions of the form $\sigma(x, y)=U(y), h(x)=1$.

The functions $\left(\sigma^{j}, \eta^{j}\right)$ can be normalized such that

$$
\int_{R} \sigma^{j} \cdot \sigma^{l}+\int_{S} \gamma_{0} \partial_{x} \eta^{j} \partial_{x} \eta^{l}=\delta_{j l}
$$

Furthermore, one verifies that all eigenvalues are negative, and orthogonality also holds with the scalar product

$$
\int_{R} A \nabla \sigma^{j} \cdot \nabla \sigma^{l}=-\lambda_{j} \delta_{j l}
$$

This scalar product defines a norm equivalent to the $H^{1}$-norm by the Poincaré inequality. We denote the Hilbert space corresponding to the product $(v, w) \mapsto \int_{R} A v \cdot w$ in the following by $L_{A}^{2}$. We next consider pairs $(u, h)=\sum_{j=1}^{\infty} c_{j}\left(\sigma^{j}, \eta^{j}\right)$. For $(u, h) \in D(B)$ we can conclude with $u_{N}:=\sum_{j=1}^{N} c_{j} \sigma^{j}$ that $\left\|\nabla u_{N}\right\|_{L_{A}^{2}} \leq\|\nabla u\|_{L_{A}^{2}}$ and

$$
\left\|\nabla \sum_{j}^{\infty} c_{j} \sigma^{j}\right\|_{L_{A}^{2}(R)}^{2}=\sum_{j}^{\infty}\left|c_{j}\right|^{2}\left|\lambda_{j}\right| .
$$

In particular, if one side in this equality is finite, then the other is also finite.

We now expand the initial values and the right-hand side in terms of eigenfunctions and write

$$
\begin{aligned}
& \left(u^{0}, h^{0}-\underline{\Delta}^{-1} f_{2}^{0}\right)=\sum_{j} a_{j}\left(\sigma^{j}, \eta^{j}\right), \quad\left(u^{1}, h^{1}-\underline{\Delta}^{-1} f_{2}^{1}\right)=\sum_{j} b_{j}\left(\sigma^{j}, \eta^{j}\right), \\
& \left(f_{0}^{0}, f_{1}^{0}-\underline{\Delta}^{-1} \frac{f_{2}^{1}-f_{2}^{0}}{\Delta t}\right)=\sum_{j} d_{j}\left(\sigma^{j}, \eta^{j}\right) .
\end{aligned}
$$

Here $\underline{\Delta}^{-1}$ denotes any right inverse of $\underline{\Delta}$. Equations (3.4), (3.5) for the first time step translate into

$$
\frac{b_{j}-a_{j}}{\Delta t}=\lambda_{j} \frac{b_{j}+a_{j}}{2}+d_{j} \quad \forall j \in \mathbb{N} .
$$


We find

$$
b_{j}=\frac{1}{\frac{1}{\Delta t}-\frac{1}{2} \lambda_{j}}\left(a_{j}\left[\frac{1}{\Delta t}+\frac{1}{2} \lambda_{j}\right]+d_{j}\right) .
$$

Therefore

$$
\frac{b_{j}-a_{j}}{\Delta t}=\frac{\lambda_{j} a_{j}+d_{j}}{1-\frac{1}{2} \lambda_{j} \Delta t}
$$

We have to estimate the $H^{1}$-norm of the function $\sum_{j} \frac{b_{j}-a_{j}}{\Delta t} \sigma_{j}$ by the $H^{1}$-norm of the function $\sum_{j}\left(\lambda_{j} a_{j}+d_{j}\right) \sigma^{j}$. We use (3.38) for the following two pairs that are both in $D(B)$ by the compatibility assumption:

$$
\begin{gathered}
\left(u^{1}-u^{0}, h^{1}-h^{0}-\underline{\Delta}^{-1}\left(f_{2}^{1}-f_{2}^{0}\right)\right) \\
\left(\nabla \cdot A \nabla u_{0}+f_{0}^{0},-a \cdot \nabla u_{0}(., 1)+f_{1}^{0}-\underline{\Delta}^{-1} \frac{f_{2}^{1}-f_{2}^{0}}{\Delta t}\right) .
\end{gathered}
$$

We can calculate

$$
\begin{aligned}
& \left\|\nabla \sum_{j} \frac{b_{j}-a_{j}}{\Delta t} \sigma_{j}\right\|_{L_{A}^{2}(R)}^{2}=\sum_{j}\left|\lambda_{j}\right|\left|\frac{b_{j}-a_{j}}{\Delta t}\right|^{2} \\
& \leq \sum_{j}\left|\lambda_{j}\right|\left|\lambda_{j} a_{j}+d_{j}\right|^{2}=\left\|\nabla \sum_{j}\left(\lambda_{j} a_{j}+d_{j}\right) \sigma_{j}\right\|_{L_{A}^{2}(R)}^{2} \\
& \leq C\left\|\nabla \cdot A \nabla u_{0}+f_{0}^{0}\right\|_{H^{1}}^{2} .
\end{aligned}
$$

This concludes the proof.

4. A discretization of the transformed equations. We perform some elementary calculations for the transformation of (2.1)-(2.3) onto a reference domain. Our aim is to replace the temperature $\Theta(t): \Omega_{t} \rightarrow \mathbb{R}$ by the new unknown $u(t): R \rightarrow \mathbb{R}$. We denote the upper boundary of $\Omega$ by $\Gamma$ and the upper boundary of $R$ by $\Gamma_{R}=\{(x, 1): x \in S\}$. Given a domain transformation $\Psi: \Omega \rightarrow R$ we use $u \circ \Psi=\Theta$ and, in the calculation below, also $v \circ \Psi=\varphi$. We define

$$
B_{i j}:=\nabla_{j} \Psi_{i}, \quad J:=\operatorname{det}(B)^{-1}, \quad A:=J \cdot B \cdot B^{t} .
$$

We see that the equation

$$
\int_{\Omega} \nabla \Theta \cdot \nabla \varphi+\int_{\Omega} f \circ \Psi \varphi-\int_{\Gamma} g \circ \Psi \varphi=0 \quad \forall \varphi \in C^{1}(\Omega)
$$

transforms into

$$
\begin{aligned}
\int_{R}\left(B^{t} \cdot \nabla u\right) \cdot\left(B^{t} \cdot \nabla v\right) J & +\int_{R} f v J \\
& -\int_{\Gamma_{R}} g v \sqrt{1+\left|\partial_{x} h\right|^{2}}=0 \quad \forall v \in C^{1}(R) .
\end{aligned}
$$


We conclude that the equation

$$
\Delta \Theta=f \circ \Psi \quad \text { in } \Omega, \quad n \cdot \nabla \Theta=g \circ \Psi \quad \text { on } \Gamma
$$

transforms into

$$
\nabla \cdot A \nabla u=J f \quad \text { in } R, \quad e_{2} \cdot A \nabla u=g \sqrt{1+\left|h^{\prime}\right|^{2}} \quad \text { on } \Gamma_{R} .
$$

Therefore the physical equations (2.1)-(2.3) transform into

$$
\begin{aligned}
J \partial_{t} u+J \partial_{t} \Psi \cdot \nabla u & =\nabla \cdot A \nabla u, \\
\partial_{t} h & =-e_{2} \cdot A \nabla u, \\
\left.u\right|_{h}+\underline{\Delta} h & =\underline{\Delta} h+\kappa(h) .
\end{aligned}
$$

The equations formally coincide with (3.1)-(3.3) if we set

$$
\begin{aligned}
& f_{0}:=(1-J) \partial_{t} u-J\left(\partial_{t} \Psi\right) \cdot \nabla u, \\
& f_{1}:=0, \quad f_{2}:=\underline{\Delta} h+\kappa(h) .
\end{aligned}
$$

We now want to choose a discretization of (4.2)-(4.4). The idea is to define matrices $A^{k}$ as in (4.1) and to define $f_{i}^{k}$ as in (4.5), (4.6). In order to proceed we have to define domain transformations $\Psi^{k}: \Omega^{k} \rightarrow R$ that we can insert in (4.1). We define $\Psi^{k}$ as the inverse of functions $X^{k}: R \rightarrow \Omega^{k}$ with

$$
X^{k+1}(x, y)-X^{k}(x, y)=\frac{X_{2}^{k}(x, y)}{h^{k}(x)}\left(h^{k+1}(x)-h^{k}(x)\right) e_{2} .
$$

We choose an initial parametrization $X^{0}$ as in Theorem 2.2.

To discretize formula (4.5) we have to discretize $\partial_{t} \Psi$. Since the definition of $X$ is consistent with the continuous equation

$$
\partial_{t} \Psi^{-1}(t, x, y)=\frac{\left(\Psi^{-1}\right)_{2}(x, y)}{h(x)} \partial_{t} h(t, x) e_{2},
$$

we find from $\partial_{t}\left(\Psi \circ \Psi^{-1}\right)=0$ the continuous equation

$$
\partial_{t} \Psi(t, \xi, \zeta)=-\partial_{\zeta} \Psi \cdot \frac{\zeta}{h} \partial_{t} h(t, \xi) .
$$

Because of $J=\left(\partial_{\zeta} \Psi_{2}\right)^{-1}$ the right-hand side of the discrete scheme can be defined consistently by

$$
\begin{aligned}
& f_{0}^{k}:=\left(1-J^{k}\right) \frac{u^{k+1}-u^{k}}{\Delta t}+\frac{X_{2}^{k}}{h^{k}} \frac{h^{k}-h^{k-1}}{\Delta t} \partial_{y} \frac{u^{k}+u^{k+1}}{2}, \\
& f_{1}^{k}:=0, \quad f_{2}^{k+1}:=\underline{\Delta} h^{k}+\kappa\left(h^{k}\right) .
\end{aligned}
$$

In the definition of $f_{0}^{0}$, the first time step, we insert the formal time derivative of $h$ instead of $\frac{h^{0}-h^{-1}}{\Delta t}$, and we use $u_{0}$ instead of $\frac{u^{0}+u^{1}}{2}$. To have $f_{2}$ defined on the whole time interval we set $f_{2}^{0}=\Delta h_{0}+\kappa\left(h_{0}\right) \equiv f_{2}^{1}$. This defines a discrete scheme that is consistent with (4.2)-(4.4). Note that in the above definition $f_{0}^{k}$ depends on $u^{k+1}$.

An assumption concerning the compatibility of the initial values will be needed. This is not an artifact of the discretization - the same is true for the continuous 
equations. Let $(u, h)$ be a classical solution such that $\partial_{t}(u, h)$ is continuous in $t=0$. We conclude that the formal time derivative $\tilde{\partial}_{t}(u, h)$ defined by (4.2) and (4.3) must satisfy on the boundary the time derivative of (4.4). We will therefore use later on the following assumption.

ASSUMPTION 4.1. We say that the compatibility conditions for the continuous equations are satisfied if for $u_{0} \in H^{3}(R)$ the formal time derivative $\tilde{\partial}_{t} u_{0}$ is in $H^{1}(R)$ and

$$
\begin{aligned}
u_{0}(., 1)-\kappa\left(h_{0}\right) & =0, \\
\tilde{\partial}_{t} u_{0}(., 1)+\underline{\Delta} \tilde{\partial}_{t} h_{0} & =0 .
\end{aligned}
$$

THEOREM 4.2. Let the initial values $\left(u_{0}, h_{0}\right)$ satisfy the compatibility condition of Assumption 4.1 and let $h_{0}-1$ be small in $C^{0,1}(S)$. We consider scheme (CN) with $f_{i}^{k}$ as in (4.7) and $A^{k}$ defined by (4.1).

Then there exists $T>0$ such that the scheme $(C N)$ has a unique solution $\left(u^{k}, h^{k}\right)_{k}$. The linear interpolants $(u, h)$ of $\left(u^{k}, h^{k}\right)_{k}$ and $\bar{u}$ of $\frac{1}{2}\left(u^{k}+u^{k+1}\right)$ satisfy the estimate

$$
\left\|\bar{\partial}_{t}(u, h)\right\|_{Y}+\left\|\partial_{x}^{2}(u, h)\right\|_{Y}+\|\bar{u}\|_{L^{\infty}\left(I ; H^{3}(R)\right)} \leq C,
$$

where $C$ and $T$ depend only on the norm of the initial values and are independent of $\Delta t$.

Proof. The proof is given in three parts (A)-(C). Part (A) is concerned with the initial values and their compatibility. In part (B) the crucial estimates on solutions are derived with the help of Proposition 3.5 on the scheme $(\mathrm{CN})$ with a fixed right-hand side. In part $(\mathrm{C})$ we show the existence of a bounded solution.

(A) Compatibility of initial values. We want to use Proposition 3.5. In order to do so, we have to guarantee that the compatibility assumption, Assumption 3.6, is satisfied. By definition of $f_{2}^{0},(3.30)$ holds. Concerning (3.31) we observe that $f_{2}^{1}-f_{2}^{0}=0$. In the above scheme the time derivative $\frac{u^{1}-u^{0}}{\Delta t}$ appears in $f_{0}^{0}$. This in general changes the compatibility condition for the scheme. However, our construction imposed $J^{0}=1$ on the upper boundary and therefore

$$
f_{0}^{0}(., 1)=-\tilde{\partial}_{t} h(0) \partial_{y} u^{0}(., 1) .
$$

Then the discrete compatibility assumption (3.31) coincides with the continuous version (4.9).

(B) Improvement of a priori bounds. This part of the proof is based on estimate (3.17). We use the constant $C_{1}$ and the first term of the right-hand side of that estimate and define

$$
C_{0}:=2 C_{1}\left[\left\|\nabla \cdot A(0) \nabla u_{0}+f_{0}(0)\right\|_{H^{1}(R)}+\left\|\partial_{x}^{2} u_{0}\right\|_{H^{1}(R)}\right] .
$$

We will show that given $\delta>0$ we can choose a small $T>0$ and a small a priori bound for $\left\|h_{0}-1\right\|_{C^{0,1}(S)}$ such that for every solution $(u, h)$

$$
\begin{aligned}
\left\|\bar{\partial}_{t}(u, h)\right\|_{Y}+\left\|\partial_{x}^{2}(u, h)\right\|_{Y} & \leq 2 C_{0} \\
\Rightarrow\left\|\bar{\partial}_{t}(u, h)\right\|_{Y}+\left\|\partial_{x}^{2}(u, h)\right\|_{Y} & \leq C_{0} .
\end{aligned}
$$

This is shown in four steps. With a constant $C$ independent of $\delta$ and $T$ there holds the following:

1. $\bar{u} \in L^{\infty}\left(I ; H^{2}(R)\right)$ is bounded by $C$.

2. The coefficients $A^{k}$ defined by (4.1) satisfy $(3.16)_{\delta}$. 
3. The norms of $\bar{\partial}_{t} f$ and of $\partial_{x}^{2} f$ on the right-hand side of (3.17) are bounded by $C \delta$.

4. The norms of $f_{0} \in L^{\infty}\left(I ; H^{1}(R)\right)$ and of $A \in L^{\infty}\left(I ; H^{2}(R)\right)$ are bounded by $C$.

Once we have shown 1-4, we can choose a new $\delta>0$ and $T>0$ and use Proposition 3.5 to obtain the implication (4.11).

Now consider a solution $(u, h)$ with the bound $2 C_{0}$ as in (4.11).

1. Regularity of $\overline{\boldsymbol{u}}$. The function $f_{0}$ is bounded in $L^{\infty}\left(I ; L^{2}(R)\right)$ (see below). We use the elliptic equation (3.4) for $u^{k+1 / 2}$ :

$$
\nabla \cdot A^{k} \nabla u^{k+1 / 2}=\frac{u^{k+1}-u^{k}}{\Delta t}-f_{0}^{k} \in L^{2}(R) .
$$

The boundary condition (3.5) is smooth enough to imply the desired estimate for $\sup _{k}\left\|u^{k+1 / 2}\right\|_{H^{2}(R)}$.

2. Estimates for $\boldsymbol{A}$. By an interpolation we see that for some $\alpha>0$ the function $h$ is also bounded as

$$
h \in C^{\alpha}\left(I ; H^{4}(S)\right)
$$

Then the matrix $B=\nabla \Psi$ satisfies

$$
B \in C^{\alpha}\left(I ; H^{3}(R)\right), \quad B(., 1) \in C^{\alpha}\left(I ; H^{3}(S)\right) .
$$

Since $H^{3}(R)$ is an algebra (see, e.g., [2]), the matrix $A$ satisfies estimates in the same spaces. Choosing $T$ small we immediately infer the first two lines in (3.16).

In order to verify the third line we again use an interpolation: with $p>2$ we find an estimate for

$$
\partial_{t} h \in L^{p}\left(I ; H^{3}(S)\right)
$$

This implies an estimate for

$$
\partial_{t} B \in L^{p}\left(I ; H^{2}(R)\right)
$$

Again, $\partial_{t} A$ satisfies estimates in the same space. If necessary we choose a smaller $T$ in order to infer the third line in (3.16).

We turn to the estimates for $f_{i}$. The function $f_{1}$ vanishes identically, and all estimates are trivial.

3. and 4. Estimates for $\boldsymbol{f}_{\mathbf{0}}$. We first consider the term $(1-J) \partial_{t} u$. The factor $(1-J)$ is small in $L^{\infty}\left(I ; L^{\infty}(R)\right)$ by smallness of $h_{0}$ in $C^{0,1}(S)$. We use

$$
\begin{aligned}
\partial_{t} u \in Y_{u} & \Rightarrow \partial_{t}^{2} u \in L^{2} L^{2} \Rightarrow \partial_{t}\left[(1-J) \partial_{t} u\right] \in L^{2} L^{2}, \\
\partial_{x}^{2} u \in Y_{u} & \Rightarrow \partial_{t} \partial_{x}^{2} u \in L^{2} L^{2} \Rightarrow \partial_{x}^{2}\left[(1-J) \partial_{t} u\right] \in L^{2} L^{2}, \\
\partial_{t} u \in Y_{u} & \Rightarrow \partial_{t} u \in L^{\infty} H^{1} \Rightarrow\left[(1-J) \partial_{t} u\right] \in L^{\infty} H^{1} .
\end{aligned}
$$

These implications together with their corresponding estimates give the desired estimate for the first term in $f_{0}$. Note that the smallness of, e.g., $\partial_{t}\left[(1-J) \partial_{t} u\right]=$ $(1-J) \partial_{t}^{2} u-\partial_{t} J \partial_{t} u$ follows for the first term by smallness of $1-J$, for the second term by a compactness argument: $\partial_{t} J \in L^{\infty} H^{3 / 2}$ and $\partial_{t} u \in L^{\infty} H^{1}$ imply (for small $T)$ smallness of the second term in $L^{2} L^{2}$. The estimate of $(1-J) \partial_{t}^{2} u$ is the only place where we use the smallness of $h_{0}$. 
The other term of $f_{0}$ has the regularity properties of $\tilde{\partial}_{t} \Psi \cdot \nabla \bar{u}$. We use step 1 with the estimate for $\bar{u} \in L^{\infty} H^{2}$. It yields

$$
\tilde{\partial}_{t} \Psi \in L^{\infty}\left(I ; H^{2+1 / 2}(R)\right), \quad \nabla \bar{u} \in L^{\infty}\left(I ; H^{1}(R)\right),
$$

and we find the estimate for $f_{0} \in L^{\infty}\left(I ; H^{1}(R)\right)$. The estimates for $\partial_{t} f_{0}$ and $\partial_{x}^{2} f_{0}$ are direct. Smallness of the $L^{2}(I)$-norms follows by the compactness argument.

Estimates for $\boldsymbol{f}_{2}$. Concerning $f_{2}$ we have to take special care of the first time step. However, let us first consider $f_{2}$ as defined by $f_{2}^{1}, \ldots, f_{2}^{K}$ : the functions $f_{2}^{k}$ are composed from first and second derivatives of $h$. Remember that the operator $-\underline{\Delta} h$ is the linearization of the mean curvature $\kappa(h)$ in $h=h_{0}$. By the $2 C_{0}$-bound of (4.11) we can estimate the differences $\partial_{x} h-\partial_{x} h_{0}$ pointwise by a small number (depending on $T)$. Then $f_{2}$ has the form $f_{2}=-\kappa(h)-\underline{\Delta} h=G\left(\partial_{x} h, \partial_{x} h_{0}\right) \cdot\left(1, \partial_{x}^{2} h\right)$ with $G(0,0)=0$ and $G$ differentiable. We find the estimate

$$
\left\|f_{2}\right\| \leq C \varepsilon\|h\|,
$$

where the norms are those of (3.17) and of (4.11), and $\varepsilon$ is arbitrarily small for $T$ small.

Let us now consider the first time step. $f_{2}^{0} \in H^{2+1 / 2}(S)$ by Assumption 4.1. There holds $f_{2}^{1}-f_{2}^{0}=0$, and we find the estimate for the first discrete time derivative of $f_{2}$. The second discrete time derivative is

$$
\bar{\partial}_{t}^{2} f_{2}(0):=\frac{f_{2}^{2}-2 f_{2}^{1}+f_{2}^{0}}{(\Delta t)^{2}}=\frac{f_{2}^{2}-f_{2}^{1}}{(\Delta t)^{2}}=\frac{\kappa\left(h^{1}\right)+\underline{\Delta} h^{1}-\kappa\left(h^{0}\right)-\underline{\Delta} h^{0}}{(\Delta t)^{2}} .
$$

We introduce $T\left[\partial_{x} h\right]:=\frac{\partial_{x} h}{\sqrt{1+\left|\partial_{x} h\right|^{2}}}$ to write

$$
\bar{\partial}_{t}^{2} f_{2}(0)=-\frac{1}{(\Delta t)^{2}} \partial_{x}\left(T\left[\partial_{x} h^{1}\right]-T\left[\partial_{x} h^{0}\right]-T^{\prime}\left[\partial_{x} h^{0}\right] \cdot \partial_{x}\left(h^{1}-h^{0}\right)\right)
$$

We find

$$
\left\|\bar{\partial}_{t}^{2} f_{2}(0)\right\|_{H^{-1}(S)} \leq C\left\|\frac{\partial_{x}\left(h^{1}-h^{0}\right)}{\Delta t}\right\|_{L^{\infty}(S)}^{2} \leq C\left\|\frac{u^{1}-u^{0}}{\Delta t}\right\|_{H^{1}(R)}^{2} .
$$

(C) Existence of a solution - the continuity argument. Note that a time step of scheme $(\mathrm{CN})$ with $f$ as in (4.7) is still a linear equation for $\left(u^{k+1}, h^{k+1}\right)$. We see that the single time step can always be solved as long as $1-J^{k}$ and $\frac{X_{2}^{k}}{h^{k}} \frac{h^{k}-h^{k-1}}{\Delta t}$ are small in $L^{\infty}$. Still, it could happen that on the time interval $\left(0, t^{k}\right)$ the solution has norm less than $C_{0}$ and on the time interval $\left(0, t^{k+1}\right)$ the solution has a norm larger than $2 C_{0}$. We will show that this cannot happen.

We connect the initial values $\left(u_{0}, h_{0}\right)$ with a continuous path $\left(u_{\lambda}, h_{\lambda}\right)_{\lambda \in[0,1]}$ with the trivial initial values $\left(u_{1}, h_{1}\right)=0$. This can be done in such a way that $\left(u_{\lambda}, h_{\lambda}\right)$ satisfies the compatibility condition for all $\lambda \in[0,1]$. If scheme $(\mathrm{CN})$ with $f$ as in (4.7) and with initial values $\left(u_{\lambda}, h_{\lambda}\right)$ has a solution on $I=(0, T)$ we denote this solution by $\left(u^{\lambda}, h^{\lambda}\right)$. This family of solutions has the following two properties.

1. Every weak $\operatorname{limit}_{\lim _{\lambda \rightarrow \lambda_{0}}}\left(u^{\lambda}, h^{\lambda}\right)$ in the topology of (4.11) of bounded solutions is again a bounded solution. This follows immediately, since we can take the limit in all equations. 
2. If $\left(u^{\lambda_{0}}, h^{\lambda_{0}}\right)$ is a solution, bounded by $C_{0}$, then also in a neighborhood $\left(\lambda_{0}-\right.$ $\varepsilon, \lambda_{0}+\varepsilon$ ) of $\lambda_{0}$ there exist solutions that are bounded by $C_{0}$. This follows because we deal with a fixed (finite) number of time steps. The norm of the solution depends continuously on $\lambda$. In general the norm might exceed the value $C_{0}$, but we can achieve that it does not exceed $2 C_{0}$. Now property (4.11) ensures that the norm remains bounded by $C_{0}$.

We combine the above facts 1 and 2 to conclude. The set

$$
\left\{\lambda \in[0,1] \mid \text { a solution }(u, h)_{\lambda} \text { exists and }\left\|(u, h)_{\lambda}\right\| \leq C_{0}\right\}
$$

is a nonempty ( $\lambda=1$ is in the set), closed (by property 1 ), and open (by property 2 ) subset of $[0,1]$. Therefore $\lambda=0$ is in the above set, and therefore a solution $\left(u^{0}, h^{0}\right)$ to initial values $\left(u_{0}, h_{0}\right)$ exists and satisfies the estimate. This concludes the proof of the theorem.

Corollary 4.3. Let $h_{0}, u_{0}$, and $T>0$ as in the last theorem. Then, for a subsequence $\Delta t \rightarrow 0$, the solutions $\left(u^{k}, h^{k}\right)$ converge to solutions of (4.2)-(4.4). In particular, (2.1)-(2.3) with compatible initial conditions possess a solution on a shorttime interval.

Proof. By the above theorem the solutions $(u, h)_{\Delta t}$ of the discrete problems are uniformly bounded. Therefore there exists a subsequence with a weak limit $(u, h)$. The convergence is strong for $u \in L^{2}\left(I ; H^{2}(R)\right) \cap H^{1}\left(I ; L^{2}(R)\right)$ and for $h \in L^{2}\left(I ; H^{5}(R)\right) \cap H^{1}\left(I ; H^{3}(R)\right)$. Because of consistency in the definition of $A$ and $f$ we can conclude that $(u, h)$ is a strong solution to the transformed equations (4.2)-(4.4). The transformed solution $(\Theta, h)$ is a solution of the original problem.

5. Proof of Theorem 2.2. Theorem 4.2 yields a stable discretization of the original equations. The drawback for a use as a numerical scheme is the need to transform all equations onto a fixed domain. It is more natural to use the operator splitting scheme (OS). We will prove in this section the stability of scheme (OS) as it was stated in Theorem 2.2. The proof uses a transformation of the discrete scheme onto a fixed domain. It will turn out that scheme (OS) is in fact identical to the scheme $(\mathrm{CN})$ of section 4.

ASSUMPTION 5.1. Let $n$ be the normal vector of the initial domain given by $h_{0}$. We introduce the formal time derivatives in $t=0$ by

$$
\begin{aligned}
\left.\tilde{\partial}_{t} \Theta\right|_{t=0} & :=\Delta \Theta_{0}, \\
\left.\tilde{\partial}_{t} h\right|_{t=0} & :=-n_{2}^{-1}\left(n \cdot \nabla \Theta_{0}\right) \circ H_{0} .
\end{aligned}
$$

We impose on the initial values the regularity $\left.\tilde{\partial}_{t} \Theta\right|_{t=0} \in H^{1}\left(\Omega_{0}\right)$ and the compatibility conditions

$$
\begin{aligned}
\Theta \circ H_{0} & =\kappa\left(h_{0}\right), \\
\left.\tilde{\partial}_{t} \Theta\right|_{t=0}\left(x, h_{0}(x)\right)+\left.\partial_{2} \Theta_{0}\left(x, h_{0}(x)\right) \cdot \tilde{\partial}_{t} h\right|_{t=0}(x) & =\left.D \kappa\left(h_{0}\right) \tilde{\partial}_{t} h\right|_{t=0}(x) .
\end{aligned}
$$

Proof of Theorem 2.2. We introduce the following functions:

$$
\begin{aligned}
& u^{k}:=\Theta^{k} \circ X^{k}: R \rightarrow \mathbb{R}, \\
& \bar{v}^{k}:=v^{k} \circ X^{k}: R \rightarrow\{0\} \times \mathbb{R} \subset \mathbb{R}^{2}, \\
& \tilde{u}^{k}:=\tilde{\Theta}^{k} \circ X^{k} .
\end{aligned}
$$


We now interpret scheme (OS) as a scheme for $\left(u^{k}, h^{k}\right)$. Step 3 of (OS) reads in the new notation

$$
\tilde{u}^{k}=u^{k+1} .
$$

We use this identity to write the equations of Step 2 in terms of $u^{k+1}$. We use the transformation of section 4 with corresponding $B^{k}, A^{k}, J^{k}$.

$$
\begin{aligned}
J^{k} \frac{u^{k+1}-u^{k}}{\Delta t}= & \nabla \cdot\left(A^{k} \nabla \frac{u^{k}+u^{k+1}}{2}\right) & & \\
& +J^{k} \bar{v}^{k} \cdot\left(B^{k}\right)^{t} \cdot \nabla \frac{u^{k}+u^{k+1}}{2} & & \text { in } R, \\
\frac{h^{k+1}-h^{k}}{\Delta t} & =-e_{2} \cdot A^{k} \cdot \nabla \frac{u^{k}+u^{k+1}}{2}(., 1) & & \text { in }[0,1], \\
u^{k+1}(., 1)+\underline{\Delta} h^{k+1} & =\kappa\left(h^{k}\right)+\underline{\Delta} h^{k} & & \text { in }[0,1] .
\end{aligned}
$$

This is nothing but scheme $(\mathrm{CN})$ with the right-hand side

$$
\begin{aligned}
& f_{0}^{k}:=\left(1-J^{k}\right) \frac{u^{k+1}-u^{k}}{\Delta t}-J^{k} \bar{v}^{k} \cdot\left(B^{k}\right)^{t} \cdot \nabla \frac{u^{k}+u^{k+1}}{2}, \\
& f_{1}^{k}:=0, \quad f_{2}^{k+1}:=\kappa\left(h^{k}\right)+\underline{\Delta} h^{k},
\end{aligned}
$$

where in the definition of $f_{0}^{0}$ the convective term is calculated explicitly. The scheme is identical to that of section 4 , since

$$
J^{k} e_{2} \cdot\left(B^{k}\right)^{t}=e_{2} \quad \text { and } \quad \bar{v}^{k}(x, y)=\frac{X_{2}^{k}(x, y)}{h^{k}(x)} \frac{h^{k}(x)-h^{k-1}(x)}{\Delta t} e_{2} .
$$

Theorem 2.2 is a consequence of Theorem 4.2.

Corollary 2.3 follows from the theorem just as Corollary 4.3 followed from Theorem 4.2. Let us demonstrate without referring to section 4 that the scheme is consistent. From (2.9) and (2.5) we conclude

$$
\Theta^{k+1} \circ X^{k+1}-\Theta^{k} \circ X^{k}=\left(\Delta \frac{\Theta^{k}+\tilde{\Theta}^{k}}{2}\right) \circ X^{k}+\left(v \cdot \nabla \frac{\Theta^{k}+\tilde{\Theta}^{k}}{2}\right) \circ X^{k} .
$$

In the limit $\Delta t \rightarrow 0$ we infer

$$
\partial_{t}(\Theta \circ X)=(\Delta \Theta) \circ X+(v \cdot \nabla \Theta) \circ X .
$$

This yields the original equation (2.1), since by definition of $v$ in (2.4)

$$
\nabla \Theta \cdot \partial_{t} X(x, y)=\nabla \Theta \cdot\left(\frac{X_{2}(x, y)}{h(x)} \partial_{t} h\right) e_{2}=v \cdot \nabla \Theta .
$$

\section{REFERENCES}

[1] F. Abergel, D. Hilhorst, F. Issard-Roch, And J. Scheid, Local existence and uniqueness of a Stefan problem with surface tension, Appl. Anal., 60 (1996), pp. 219-240.

[2] R.A. Adams, Sobolev Spaces, Pure Appl. Math. 65, Academic Press, New York, 1975.

[3] E. BÄNSCH, Numerical Methods for the Instationary Navier-Stokes Equations with a Free Capillary Surface, Habilitationsschrift, Universität Freiburg, 1998. 
[4] X. Chen And F. Reitich, Local existence and uniqueness of solutions of the Stefan problem with surface tension and kinetic undercooling, J. Math. Anal. Appl., 164 (1992), pp. 350362.

[5] J. CRANK AND P. Nicolson, A practical method for numerical evaluation of solutions of partial differential equations of the heat-conduction type, Proc. Cambridge Philos. Soc., 43 (1947), pp. 50-67.

[6] Sт. Luckhaus, Solutions for the two-phase Stefan problem with the Gibbs-Thomson law for the melting temperature, European J. Appl. Math., 1 (1990), pp. 101-111.

[7] A.M. Meirmanov, The Stefan problem with surface tension in the three dimensional case with spherical symmetry: Nonexistence of the classical solution, European J. Appl. Math., 5 (1994), pp. 1-19.

[8] E.V. RADKEVICH, On conditions for the existence of a classical solution of the modified Stefan problem (the Gibbs-Thompson law), Math. USSR-Sb., 75 (1993), pp. 221-246.

[9] B. SchweIzer, Free boundary fluid systems in a semigroup approach and oscillatory behavior, SIAM J. Math. Anal., 28 (1997), pp. 1135-1157.

[10] A. VeEser, Error estimates for semi-discrete dendritic growth, Interfaces Free Bound., 1 (1999), pp. 227-255.

[11] A. Visintin, Models of Phase Transitions, Progr. Nonlinear Differential Equations Appl. 28, Birkhäuser, Boston, 1996. 